\title{
High-order unidirectional model with adjusted coefficients for large-amplitude long internal waves
}

Wooyoung Choi

Dept. of Mathematical Sciences, New Jersey Institute of Technology, Newark, NJ 07102, USA

Changhong Zhi

School of Naval Arch., Ocean and Civil Eng., Shanghai Jiao Tong University, Shanghai, China

Ricardo Barros

Dept. of Mathematical Sciences, Loughborough University, Loughborough, LE11 3TU, UK

May 26, 2020

\begin{abstract}
To describe large amplitude internal solitary waves in a two-layer system, we consider the high-order unidirectional (HOU) model that extends the Korteweg-de Vries equation with high-order nonlinearity and leading-order nonlinear dispersion. While the original HOU model is valid only for weakly nonlinear waves, its coefficients depending on the depth and density ratios are adjusted such that the adjusted model can represent the main characteristics of large amplitude internal solitary waves, including effective wavelength, wave speed, and maximum wave amplitude. It is shown that the solitary wave solution of the adjusted HOU (aHOU) model agrees well with that of the strongly nonlinear MiyataChoi-Camassa (MCC) model up to the maximum wave amplitude, which cannot be achieved by the original HOU model. To further validate the aHOU model, numerical solutions of the aHOU model are presented for the propagation and interaction of solitary waves and are shown to compare well with those of the MCC model. The aHOU model is further extended to the case of variable bottom and and is solved numerically. In comparison with the MCC model for variable bottom, it is found that the aHOU model is a simple, but reliable theoretical model for large amplitude internal solitary waves, which would be useful for practical applications.
\end{abstract}

\section{Introduction}

Large amplitude internal solitary waves in density-stratified oceans are typically generated by tidal flows over topographical disturbances and have been observed via satellite images as well as field experiments. Due to the reduced effective gravity by small density variation in the ocean, the amplitudes of the internal solitary waves can be as large as the characteristic length scale in the vertical direction, such as the thickness of the mixed layer, which could be on the order of $100 \mathrm{~m}$ (Helfrich \& Melville, 2006). Therefore, it is non-trivial to describe the large 
amplitude wave motions using a simple theoretical model developed under the small amplitude assumption.

For a two-layer system, a theoretical model for large amplitude long internal waves has been proposed by Miyata (1987) and Choi \& Camassa (1999) and the validity of this strongly nonlinear model, often referred to as the MCC model, has been confirmed through the comparison of its solitary wave solution with the numerical solution of the Euler equations and laboratory measurements (Camassa et al. 2006; Kodaira et al. 2016). However the application of the MCC model to unsteady wave problems could be problematic as its initial value problem is ill-posed due to the local Kelvin-Helmholtz instability excited by a velocity jump across the deformed interface (Jo \& Choi 2002). While such shear instability persists in laboratory and field experiments, it has been observed that only relatively large amplitude waves are deformed by the instability. Even when they are unstable, the solitary waves of large amplitudes maintain approximately their profiles although dispersive tails excited by Kelvin-Helmholtz billows are observed (Grue et al. al 1999; Kodaira et al. 2016). Therefore stabilizing effects induced by, for example, viscosity and a thin density transition layer need to be incorporated into the MCC model for unsteady problems. Unfortunately, modeling these stabilizing effects in an asymptotically-consistent way is difficult, particularly, under the strongly nonlinear assumption.

Numerically the unsteady MCC model has been solved with a low-pass filter to remove unstable short waves (Jo \& Choi 2008). This numerical approach was justified by the fact that the MCC model is valid only for long waves and unstable short waves described incorrectly by the long wave model should be filtered out. The filter was successfully used for the propagation of a single solitary wave, where the critical wave number beyond which the instability is observed can be predicted. For more general problems, the filter might remove too much or too little energy. As an alternative approach, the MCC model was regularized (Choi et al. 2009) by changing the dispersive behavior of the model with adopting, instead of the depth-averaged horizontal velocities, the horizontal velocities at certain vertical levels as dependent variables. While an efficient iterative scheme to solve the regularized model was proposed (Choi et al. 2011), it still requires a numerical parameter to be tuned for faster convergence of their iterative scheme. Furthermore, to solve numerically the MCC model, one should know initially not only the interface displacement, but also the initial velocity fields that might be unavailable. Therefore, for practical applications, it would be desirable if one could find a relatively simple, but reliable model that describes the evolution of large amplitude internal solitary waves as accurately as the MCC model.

Previously there have been numerous attempts to improve the well-known Korteweg-de Vries $(\mathrm{KdV})$ equation for weakly nonlinear unidirectional waves. At the critical depth ratio, where the coefficient for the second-order nonlinear term in the KdV equation vanishes, the modified KdV equation with a third-order nonlinear term can be obtained. Near this criticality, both second- and third-order terms are necessary along with the linear dispersive term in the KdV equation, which yields the so-called Gardner equation (Michallet \& Barthélemy, 1998; Grimshaw et al., 2002). By breaking the balance between nonlinearity and dispersion to describe the strong nonlinearity of large amplitude long internal waves, a high-order unidirectional (HOU) model has been proposed by Choi \& Camassa (1999). A related model, although more phenomenological, has been proposed by Ostrovsky \& Grue (2003) by replacing the linear wave speed and the undisturbed layer thicknesses in the dispersive term in the KdV equation by the 


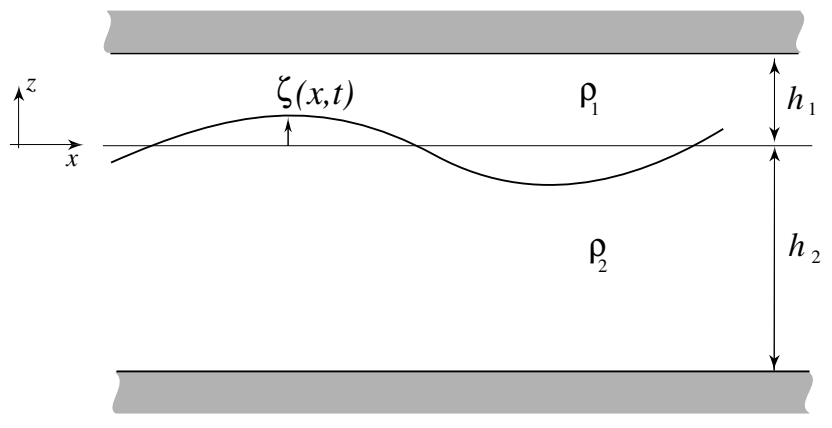

Figure 1: Two-fluid system

characteristic speed of the shallow water equations and the local layer thicknesses, respectively. In this paper, the HOU model is modified by adjusting its coefficients so that the adjusted HOU (aHOU) model preserves the solitary wave characteristics of the MCC model. To show that the good agreement observed for steady solutions persists for time-dependent problems, the unsteady aHOU model is solved numerically and is compared with the MCC model.

After reviewing in $\S 2$ the strongly nonlinear MCC model and weakly-nonlinear unidirectional models for internal waves, the aHOU model is proposed and its solitary wave solutions are discussed in comparison with those of the MCC model in $\S 3$. Then the aHOU model is further generalized for variable bottom and its numerical solutions are presented in $\S 4$. Concluding remarks are given in $\S 5$.

\section{Nonlinear long wave models}

\subsection{Strongly nonlinear MCC equations}

In a system of two homogeneous liquid layers of densities $\rho_{i}$ and undisturbed thicknesses $h_{i}$ $(i=1,2)$ with $\rho_{2}>\rho_{1}$ for stable stratification, the interface displacement $\zeta(x, t)$ and the depthaveraged velocities $\bar{u}_{i}(x, t)$ can be described by a strongly nonlinear long wave model (Miyata 1987; Choi \& Camassa 1999), commonly referred to as the Miyata-Choi-Camassa (MCC) model, given by

$$
\eta_{i t}+\left(\eta_{i} \bar{u}_{i}\right)_{x}=0, \quad \bar{u}_{i t}+\bar{u}_{i} \bar{u}_{i x}+g \zeta_{x}=-\frac{P_{x}}{\rho_{i}}+\frac{1}{\eta_{i}}\left(\frac{1}{3} \eta_{i}{ }^{3} G_{i}\right)_{x}+O\left(\epsilon^{4}\right),
$$

where subscripts with respect to space $x$ and time $t$ represent partial differentiation. In (2.1), $g$ is the gravitational acceleration, $P$ is the pressure at the interface, $\eta_{i}(i=1,2)$ are the local layer thicknesses defined by $\eta_{1}=h_{1}-\zeta$ and $\eta_{2}=h_{2}+\zeta$, and $G_{i}(i=1,2)$ representing the linear and nonlinear dispersive effects are given by

$$
G_{i}(x, t)=\bar{u}_{i x t}+\bar{u}_{i} \bar{u}_{i x x}-\left(\bar{u}_{i x}\right)^{2} .
$$

This model assumes $h_{2} / h_{1}=O(1)$ and is valid to $O\left(\epsilon^{2}\right)$, where $\epsilon=h_{1} / \lambda \ll 1$ with $\lambda$ being the characteristic wavelength is the dispersion parameter. On the other, the nonlinear parameter 
defined by $\alpha=a / h_{1}$ that is often assumed small for weakly nonlinear waves is no longer small and is assumed $O(1)$. Therefore, the system given by (2.1) is considered a strongly nonlinear long wave model.

For traveling-wave solutions of (2.1), after assuming $\zeta=\zeta(X), \bar{u}_{i}=\bar{u}_{i}(X)$, and $P=P(X)$ with $X=x-c t$ with $c$ being the wave speed, and imposing $\zeta, \zeta_{X}, \zeta_{X X} \rightarrow 0, \bar{u}_{i} \rightarrow 0, P_{X} \rightarrow 0$ at infinity, the system given by (2.1) can be integrated (Choi \& Camassa 1999) to

$$
\zeta_{X}^{2}=q_{0} \frac{\zeta^{2}\left(\zeta^{2}+q_{1} \zeta+q_{2}\right)}{\zeta-a_{*}}=q_{0} \frac{\zeta^{2}\left(\zeta-a_{-}\right)\left(\zeta-a_{+}\right)}{\zeta-a_{*}},
$$

where $a_{*}$ and $q_{i}(i=0,1,2)$ are given by

$a_{*}=-\frac{h_{1} h_{2}\left(\rho_{1} h_{2}+\rho_{2} h_{1}\right)}{\rho_{1} h_{1}^{2}-\rho_{2} h_{2}^{2}}, \quad q_{0}=\frac{3 g\left(\rho_{2}-\rho_{1}\right)}{c^{2}\left(\rho_{1} h_{1}^{2}-\rho_{2} h_{2}^{2}\right)}, \quad q_{1}=-\frac{c^{2}}{g}-h_{1}+h_{2}, \quad q_{2}=h_{1} h_{2}\left(\frac{c^{2}}{c_{0}^{2}}-1\right)$,

with the solitary wave speed $c$ and the linear long wave speed $c_{0}$ given by

$$
c^{2}=c_{0}^{2}\left[\frac{\left(h_{1}-a\right)\left(h_{2}+a\right)}{h_{1} h_{2}-\left(c_{0}^{2} / g\right) a}\right], \quad c_{0}^{2}=\frac{g h_{1} h_{2}\left(\rho_{2}-\rho_{1}\right)}{\rho_{1} h_{2}+\rho_{2} h_{1}} .
$$

As shown in Choi \& Camassa (1999), either $a_{-}$or $a_{+}$is the wave amplitude depending on the density and depth ratios. In agreement with the solitary-wave solutions for the Euler equations, the traveling waves are symmetric and the sign of their amplitude $a$ is determined by the sign of the quantity $\rho_{1} h_{2}^{2}-\rho_{2} h_{1}^{2}$. More specifically, waves are of elevation (or depression) if $\rho_{1} h_{2}^{2}-\rho_{2} h_{1}^{2}$ is negative (or positive). Moreover, in the critical case when $\rho_{1} h_{2}^{2}=\rho_{2} h_{1}^{2}$, no solitary wave solutions exist (Craig \& Sterberg 1992).

When $a_{-}=a_{+}=a_{m}$, the solitary wave has the maximum amplitude and becomes a front wave (or internal bore), whose amplitude $a_{m}$ and speed $c_{m}$ are given by

$$
a_{m}=\frac{h_{1}-h_{2} \sqrt{\rho_{1} / \rho_{2}}}{1+\sqrt{\rho_{1} / \rho_{2}}}, \quad c_{m}^{2}=g\left(h_{1}+h_{2}\right) \frac{1-\sqrt{\rho_{1} / \rho_{2}}}{1+\sqrt{\rho_{1} / \rho_{2}}} .
$$

Notice that $a_{m}$ and $c_{m}$ are identical to the amplitude and speed of the internal bore for the Euler equations (Choi \& Camassa 1999).

\section{$2.2 \quad$ High-order unidirectional model}

For unidirectional waves of relatively large amplitudes, one can imagine that the KdV equation needs to be improved by including higher-order nonlinear and dispersive terms. To obtain such a model, instead of $\alpha=O\left(\epsilon^{2}\right) \ll 1$ valid for the KdV equation, the balance between nonlinearity and dispersion might need to be altered. Under the assumption of $\epsilon^{2}<\alpha<\epsilon$ (Choi \& Camassa 1999, Appendix A), the MCC equations (2.1) can be reduced, for the interface elevation $\zeta(x, t)$, to

$$
\zeta_{t}+c_{0} \zeta_{x}+c_{1} \zeta \zeta_{x}+c_{2} \zeta_{x x x}+c_{3} \zeta^{2} \zeta_{x}+\left(c_{4} \zeta_{x}^{2}+c_{5} \zeta \zeta_{x x}\right)_{x}=O\left(\epsilon^{4}, \alpha^{3}, \alpha^{2} \epsilon^{2}\right),
$$

where $c_{i}(i=1, \cdots, 5)$ are given by

$$
c_{1}=-\frac{3 c_{0}}{2} \frac{\rho_{1} h_{2}^{2}-\rho_{2} h_{1}^{2}}{\rho_{1} h_{1} h_{2}^{2}+\rho_{2} h_{1}^{2} h_{2}}, c_{2}=\frac{c_{0}}{6} \frac{\rho_{1} h_{1}^{2} h_{2}+\rho_{2} h_{1} h_{2}^{2}}{\rho_{1} h_{2}+\rho_{2} h_{1}}, c_{3}=\frac{7 c_{1}^{2}}{6 c_{0}}-\frac{3 c_{0}\left(\rho_{1} h_{2}^{3}+\rho_{2} h_{1}^{3}\right)}{h_{1}^{2} h_{2}^{2}\left(\rho_{1} h_{2}+\rho_{2} h_{1}\right)},
$$




$$
c_{4}=\frac{17 c_{1} c_{2}}{12 c_{0}}-\frac{c_{0} h_{1} h_{2}\left(\rho_{2}-\rho_{1}\right)}{12\left(\rho_{1} h_{2}+\rho_{2} h_{1}\right)}, \quad c_{5}=\frac{7 c_{1} c_{2}}{3 c_{0}}-\frac{c_{0} h_{1} h_{2}\left(\rho_{2}-\rho_{1}\right)}{6\left(\rho_{1} h_{2}+\rho_{2} h_{1}\right)} .
$$

Notice that $c_{2} / c_{0}>0$ and $c_{3} / c_{0}<0$ for all physical parameters $\left(\rho_{2} / \rho_{1}, h_{2} / h_{1}\right)$ while the signs of $c_{i} / c_{0}$ for $i=1,4,5$ can vary depending on the physical parameters. The errors in (2.11) represent those relative to the leading-order term $\zeta_{t}=O\left(\zeta_{x}\right)$. When the nonlinear dispersive terms (with $c_{4}$ and $c_{5}$ ) are neglected, (2.7) can be reduced to the Gardner equation:

$$
\zeta_{t}+c_{0} \zeta_{x}+c_{1} \zeta \zeta_{x}+c_{2} \zeta_{x x x}+c_{3} \zeta^{2} \zeta_{x}=O\left(\alpha \epsilon^{2}, \epsilon^{4}\right)
$$

Previously, (2.10) has been obtained e.g. by Kakutani \& Yamazaki (1978) for internal waves under the criticality condition of $h_{2} / h_{1}=\left(\rho_{2} / \rho_{1}\right)^{1 / 2}$ for which $c_{1}$ vanishes or is small. Here no such assumption has been made and, therefore, the coefficients given by (2.8) are slightly different from those of Kakutani \& Yamazaki (1978). They match, however, with the first four coefficients for higher-order extension of the KdV equation presented by Koop \& Butler (1981, Appendix A). When the third-order nonlinear term $\left(c_{3}=0\right)$ is neglected from the Gardner equation, the well-known $\mathrm{KdV}$ equation is recovered.

While (2.7) conserves excess mass, or $\int \zeta \mathrm{d} x$, it possesses no energy-like conservative quantity. In Choi \& Camassa (1999, Appendix A), an alternative form was suggested with the same order of approximation as in (2.7). Using $\zeta_{x x t}+c_{0} \zeta_{x x x}+c_{1}\left(\zeta \zeta_{x}\right)_{x x}=O\left(\epsilon^{4}\right)$, equation (2.7) is then modified to

$$
\zeta_{t}+c_{0} \zeta_{x}+c_{1} \zeta \zeta_{x}+\frac{c_{2}}{2} \zeta_{x x x}-\frac{c_{2}}{2 c_{0}} \zeta_{x x t}+c_{3} \zeta^{2} \zeta_{x}+\left(c_{5}-c_{4}\right)\left(\zeta_{x}^{2}+2 \zeta \zeta_{x x}\right)_{x}=0,
$$

where $\left(c_{5}-2 c_{4}\right) / c_{1}=-c_{2} /\left(2 c_{0}\right)$ has been used. This particular form to be hereafter called the High-Order Unidirectional (HOU) model conserves the following energy-like quantity $\mathcal{E}_{\mathrm{HOU}}$

$$
\mathcal{E}_{\mathrm{HOU}}=\frac{1}{2} \int_{-\infty}^{\infty}\left[\zeta^{2}+\left(\frac{c_{2}}{2 c_{0}}\right) \zeta_{x}^{2}\right] \mathrm{d} x
$$

The HOU model can be easily integrated twice, for waves propagating with the wave speed $c$, to

$$
\zeta_{X}^{2}=\tilde{q}_{0} \frac{\zeta^{2}\left(\zeta^{2}+\tilde{q}_{1} \zeta+\tilde{q}_{2}\right)}{\zeta-\tilde{a}_{*}}=\tilde{q}_{0} \frac{\zeta^{2}\left(\zeta-\tilde{a}_{-}\right)\left(\zeta-\tilde{a}_{+}\right)}{\zeta-\tilde{a}_{*}}, \quad X=x-c t,
$$

where $\tilde{q}_{i}(i=0,1,2)$ and $\tilde{a}_{*}$ are given by

$$
\tilde{q}_{0}=\frac{c_{3}}{12\left(c_{4}-c_{5}\right)}, \quad \tilde{q}_{1}=2 c_{1} / c_{3}, \quad \tilde{q}_{2}=-6\left(c-c_{0}\right) / c_{3}>0, \quad \tilde{a}_{*}=-\frac{c_{2}\left(c / c_{0}+1\right)}{2\left(c_{4}-c_{5}\right)} .
$$

As $c / c_{0}>1$ and $c_{3} / c_{0}<0, \tilde{q}_{2}$ is always positive and, therefore, the polarity of the solitary wave solution depends on the sign of $\tilde{q}_{1}$, i.e., on the sign of $\rho_{1} h_{2}^{2}-\rho_{2} h_{1}^{2}$, in agreement with the strongly and fully nonlinear theories. Therefore, if $c_{1} / c_{0}$ is positive, $\tilde{q}_{1}<0$ and the amplitude of the solitary wave is given by $a=\tilde{a}_{+}<0$ with $\tilde{a}_{-}<\tilde{a}_{+}<0$. On the other hand, if $c_{1} / c_{0}$ is negative, $\tilde{q}_{1}>0$ and the amplitude is given by $a=\tilde{a}_{-}>0$ with $0<\tilde{a}_{-}<\tilde{a}_{+}$. 


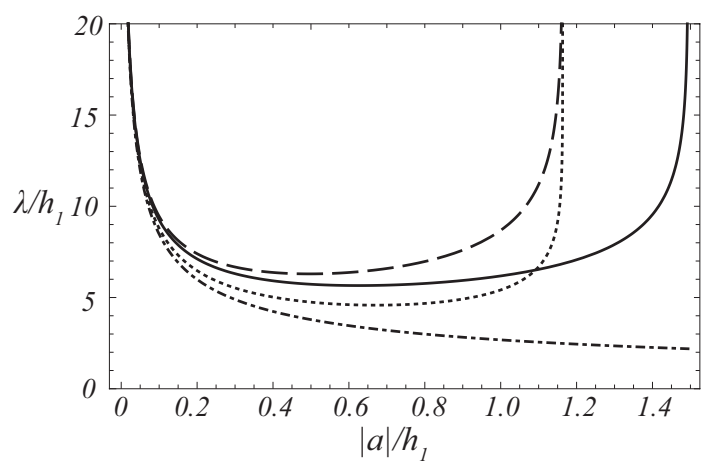

$(a)$

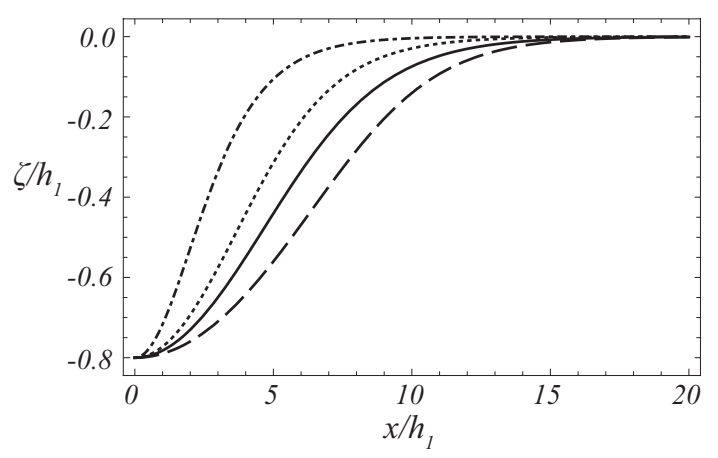

(b)

Figure 2: Comparison between models for $\rho_{2} / \rho_{1}=1.01$ and $h_{2} / h_{1}=4$. (a) Effective wavelength $\left(\lambda / h_{1}\right)$ versus wave amplitude $\left(a / h_{1}\right)$; $(b)$ Half profiles for a solitary wave of depression of $a / h_{1}=-0.8$. Solid: MCC; long-dashed: HOU; dotted: Gardner; dot-dashed: KdV.

Similarly to the MCC equations, from the condition $\zeta_{X}=0$ at $\zeta=a$, the right-hand side of (2.13) yields the following relationship between wave speed and amplitude for the HOU model:

$$
c_{\mathrm{HOU}}=c_{0}+\frac{c_{1} a}{3}\left(1+\frac{a c_{3}}{2 c_{1}}\right) .
$$

When $\tilde{a}_{-}=\tilde{a}_{+}=\tilde{a}_{m}$, the solitary wave becomes the internal bore, whose amplitude $\tilde{a}_{m}$ and speed $\tilde{c}_{m}$ are given by

$$
\tilde{a}_{m}=-\tilde{q}_{1} / 2=-c_{1} / c_{3}, \quad \tilde{c}_{m}=c_{0}-c_{1}^{2} /\left(6 c_{3}\right) .
$$

It is interesting to notice that the mathematical structure of (2.13) is identical to that of the strongly nonlinear MCC equations for traveling waves given by (2.3). Furthermore, the HOU model (2.11) describes physical processes relevant for large amplitude waves, including the higher-order nonlinear and leading-order nonlinear dispersive effects through the terms proportional to $c_{3}$ and $c_{4}-c_{5}$, respectively.

\subsection{Limitations of weakly nonlinear unidirectional models}

The comparison between the solitary wave solutions of the MCC, HOU, Gardner, and KdV equations is shown in figure 2 for fixed parameters $\rho_{2} / \rho_{1}=1.01$ and $h_{2} / h_{1}=4$, relevant to ocean applications. In addition to the half solitary wave profile for $a / h_{1}=-0.8$, we compute the effective wavelength $\lambda$ defined (Koop \& Butler 1981) by

$$
\lambda \equiv\left|\frac{1}{a} \int_{0}^{\infty} \zeta(X) \mathrm{d} X\right|=\left|\frac{1}{a} \int_{0}^{\infty} \frac{\zeta}{(\mathrm{d} \zeta / \mathrm{d} X)} \mathrm{d} \zeta\right|
$$

As shown in figure 2(a), the KdV equation is valid only for small amplitude waves. On the other hand, the Gardner and HOU models improve agreement with MCC for moderate wave amplitudes, but fail to describe large internal solitary waves, as shown in figure $2(b)$. For the 
physical parameters chosen for figure 2, notice that the Gardner and HOU models share the same maximum amplitude, but its value is much less than that of the MCC equations (and the Euler equations).

Under usual oceanic conditions, where internal solitary waves are typically of depression with $h_{2} / h_{1}>\left(\rho_{2} / \rho_{1}\right)^{1 / 2}$, one can conclude that both HOU and Gardner models under-predict the maximum wave amplitude. On the other hand, the weakly nonlinear models can overestimate the maximum wave amplitude for internal waves of elevation. Either way, the weakly nonlinear uni-directional models are not reliable to describe large amplitude internal solitary waves of interest. This is not so surprising as the unidirectional models should be valid only for weakly nonlinear waves.

Here we attempt to find a unidirectional model that is relatively simple, but is applicable to a wide range of wave amplitudes, including those close to the maximum. Particularly the mathematical structure of the HOU model is appealing as it contains terms describing the characteristics of large amplitude waves, such as higher-order nonlinearity and nonlinear dispersion. Therefore, if modified appropriately, the HOU model could serve as a useful theoretical tool for practical applications.

\section{The HOU model with adjusted coefficients}

To describe large amplitude internal solitary waves, the coefficients of the HOU model given by (2.11) are replaced by unspecified coefficients:

$$
\zeta_{t}+\mu_{0} \zeta_{x}+\mu_{1} \zeta \zeta_{x}+\mu_{21} \zeta_{x x x}-\mu_{22} \zeta_{x x t}+\mu_{3} \zeta^{2} \zeta_{x}+\mu_{4}\left(\zeta_{x}^{2}+2 \zeta \zeta_{x x}\right)_{x}=0,
$$

where $\mu_{i}$ are the coefficients to be determined. In (3.1), the coefficients for the linear terms $\left(\mu_{0}, \mu_{21}\right.$, and $\left.\mu_{22}\right)$ would be chosen to better represent the linear dispersive behavior of linear long waves while $\mu_{1}$ and $\mu_{3}$ will be determined to better describe the nonlinear effects of large amplitude internal solitary waves. On the other hand, $\mu_{4}$ will be determined to account for the nonlinear dispersive effects.

By rewriting (3.1) as

$$
\left(\zeta-\mu_{22} \zeta_{x x}\right)_{t}+\left[\mu_{0} \zeta+\frac{1}{2} \mu_{1} \zeta^{2}+\mu_{21} \zeta_{x x}+\frac{1}{3} \mu_{3} \zeta^{3}+\mu_{4}\left(\zeta_{x}^{2}+2 \zeta \zeta_{x x}\right)\right]_{x}=0,
$$

one can see that the adjusted HOU (aHOU) model given by (3.1) or (3.2) conserves not only excess mass $\int \zeta \mathrm{d} x$, but also an energy-like quantity given by

$$
\mathcal{E}_{\text {aHOU }}=\frac{1}{2} \int_{-\infty}^{\infty}\left(\zeta^{2}+\mu_{22} \zeta_{x}^{2}\right) \mathrm{d} x .
$$

To find its solitary wave solution, (3.2) can be integrated into

$$
\zeta_{X}^{2}=\gamma_{0} \frac{\zeta^{2}\left(\zeta^{2}+\gamma_{1} \zeta+\gamma_{2}\right)}{\zeta-\bar{a}_{*}}
$$

where $\gamma_{0}$ and $\bar{a}_{*}$ are given by

$$
\gamma_{0}=-\frac{\mu_{3}}{12 \mu_{4}}, \quad \gamma_{1}=\frac{2 \mu_{1}}{\mu_{3}}, \quad \gamma_{2}=-\frac{6\left(c-\mu_{0}\right)}{\mu_{3}} . \quad \bar{a}_{*}=-\frac{\mu_{21}+c \mu_{22}}{2 \mu_{4}} .
$$


From $\zeta_{X}=0$ with $\zeta=a$, the wave speed of the solitary wave for the aHOU equation can be obtained, from (3.4), as

$$
c_{\mathrm{aHOU}}=\mu_{0}+\frac{1}{6}\left(2 \mu_{1} a+\mu_{3} a^{2}\right) .
$$

The maximum amplitude $\bar{a}_{m}=-\gamma_{1} / 2$ and the corresponding wave speed $\bar{c}_{m}$ are given, from (3.6), by

$$
\bar{a}_{m}=-\frac{\mu_{1}}{\mu_{3}}, \quad \bar{c}_{m}=\mu_{0}-\frac{\mu_{1}^{2}}{6 \mu_{3}} .
$$

\subsection{Choice of coefficients}

Now we need to fix the coefficients $\left(\mu_{i}\right)$ as functions of the density and depth ratios such that the aHOU equation approximates well its parent equations, which should be the Euler equations. However, as no analytic solutions are available for the Euler equations and the MCC solutions agree well with the Euler solutions for large amplitude long waves, we develop a model that approximates the MCC model. Then we choose $\mu_{i}$ such that the MCC and aHOU equations share the main characteristics of large amplitude internal solitary waves.

We first fix $\mu_{0}, \mu_{21}$, and $\mu_{22}$ to better match the linear dispersion relation with that of the Euler equations. When linearized, the aHOU model has the linear wave speed $c_{\text {lin }}^{\text {aHou }}$ given by

$$
c_{\mathrm{lin}}^{\mathrm{aHOU}}=\frac{\mu_{0}-\mu_{21} k^{2}}{1+\mu_{22} k^{2}}=\mu_{0}-\left(\mu_{21}+c_{0} \mu_{22}\right) k^{2}+\left(\mu_{21}+c_{0} \mu_{22}\right) \mu_{22} k^{4}+O\left(k^{6}\right),
$$

where $k$ is assumed to be small for long waves. This wave speed can be matched, up to $O\left(k^{4}\right)$, with the exact linear wave speed given by

$$
c_{\text {lin }}^{\text {Euler }}=\left[\frac{(g / k)\left(\rho_{2}-\rho_{1}\right)}{\rho_{1} \operatorname{coth} k h_{1}+\rho_{2} \operatorname{coth} k h_{2}}\right]^{1 / 2}=c_{0}-c_{2} k^{2}+c_{6} k^{4}+O\left(k^{6}\right),
$$

where $c_{6}$ is given by

$$
c_{6}=\frac{3 c_{2}^{2}}{2 c_{0}}+\frac{c_{0} h_{1} h_{2}\left(\rho_{1} h_{1}^{3}+\rho_{2} h_{2}^{3}\right)}{90\left(\rho_{1} h_{2}+\rho_{2} h_{1}\right)} .
$$

From (3.8) and (3.9), one can choose $\mu_{0}, \mu_{21}$, and $\mu_{22}$ as

$$
\mu_{0}=c_{0}, \quad \mu_{21}=c_{2}-c_{0}\left(c_{6} / c_{2}\right), \quad \mu_{22}=c_{6} / c_{2},
$$

which is equivalent to the Pade $(2,2)$ approximation to the full linear dispersion relation given by (3.9). Compared with the wave speed of the original HOU model given by

$$
c_{\mathrm{lin}}^{\mathrm{HOU}}=\frac{c_{0}-\left(c_{2} / 2\right) k^{2}}{1+\left(c_{2} /\left(2 c_{0}\right)\right) k^{2}}=c_{0}-c_{2} k^{2}+\left(c_{2}^{2} /\left(2 c_{0}\right)\right) k^{4}+O\left(\epsilon^{6}\right),
$$

the wave speed given by (3.8) with (3.11) better approximates that of the Euler equations given by (3.9). Alternatively, $\mu_{21}$ and $\mu_{22}$ can be adjusted to match the linear wave speed of the MCC model, but this has not been pursued here as the MCC model approximates the linear long wave speed of the Euler equations less accurately than the aHOU model. 


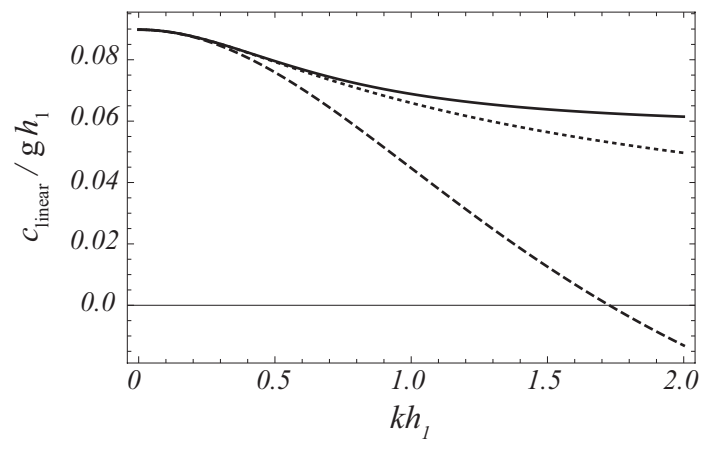

$(a)$

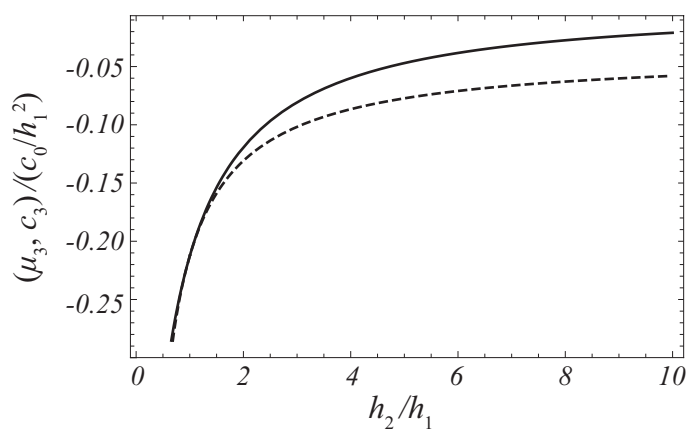

$(c)$

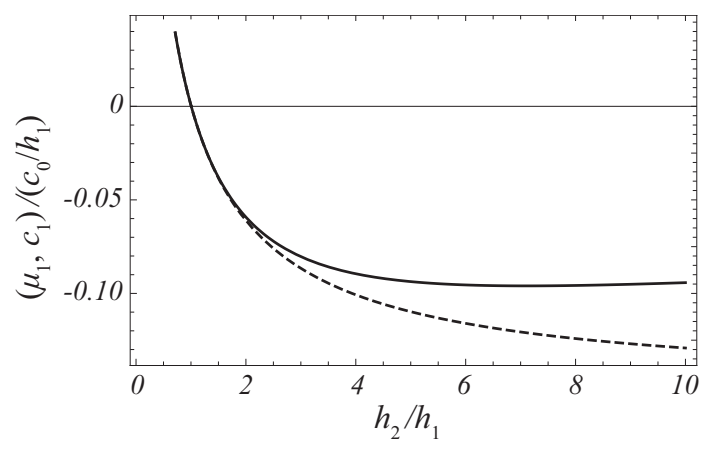

(b)

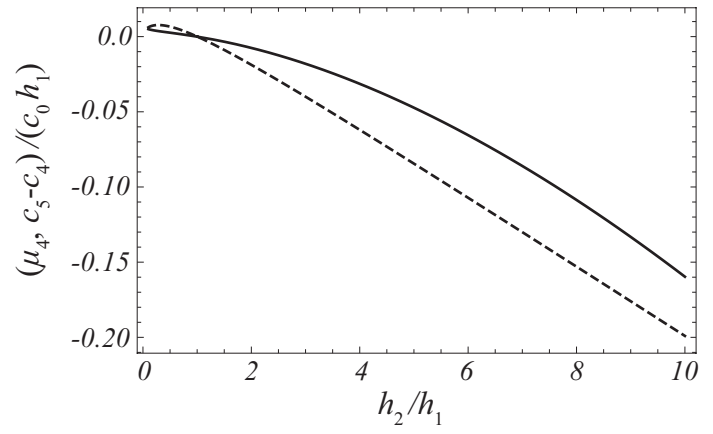

$(d)$

Figure 3: Comparison between the aHOU (solid) and HOU (dashed) models: $(a)$ linear wave speed $c_{\text {lin }}$ along with that of the linearized Euler equations (dotted); $(b)$ coefficient of the quadratic nonlinear term: $\mu_{1}$ (solid) and $c_{1}$ (dashed); $(c)$ coefficient of the cubic nonlinear term: $\mu_{3}$ (solid) and $c_{3}$ (dashed); $(d)$ coefficient of the nonlinear dispersive term: $\mu_{4}$ (solid) and $c_{5}-c_{4}$ (dashed). Here the density ratio is set as $\rho_{2} / \rho_{1}=1.01$.

Next we choose $\mu_{1}$ and $\mu_{3}$ such that the maximum wave amplitude and the maximum wave speed of the aHOU equation given by (3.7) match those of the MCC model given by (2.6). This can be achieved by replacing $\bar{a}_{m}$ and $\bar{c}_{m}$ in (3.7) by $a_{m}$ and $c_{m}$ in (2.6), respectively, which yields the expressions of $\mu_{1}$ and $\mu_{3}$, in terms of $a_{m}$ and $c_{m}$, as

$$
\mu_{1}=6\left(c_{m}-c_{0}\right) / a_{m}, \quad \mu_{3}=-6\left(c_{m}-c_{0}\right) / a_{m}^{2} .
$$

As shown in (2.6), $c_{m}$ and $a_{m}$ are functions of the density and depth ratios, $\rho_{2} / \rho_{1}$ and $h_{2} / h_{1}$, and, therefore, so are $\mu_{1}$ and $\mu_{3}$.

Finally the coefficient of nonlinear dispersive terms $\mu_{4}$ is chosen by matching the characteristic length scale of the solitary wave solution at the maximum amplitude. By comparing (2.3) and (3.4), one can see that this is equivalent to imposing the condition of $\gamma_{0}=q_{0}$ with $q_{0}$ given by (2.4) being evaluated with $a=a_{m}$ and $c=c_{m}$. Then the expression of $\mu_{4}$ is found as

$$
\mu_{4}=\frac{\left(\rho_{1} h_{1}^{2}-\rho_{2} h_{2}^{2}\right)}{6 g\left(\rho_{2}-\rho_{1}\right)} \frac{c_{m}^{2}\left(c_{m}-c_{0}\right)}{a_{m}^{2}} .
$$


With this choice of $\mu_{4}$, the effective wavelength is expected to be better predicted.

Figure 3 shows the comparison of the linear wave speed and the coefficients between the original HOU model and the aHOU model. As shown in figure 3(a), the linear wave speed of the aHOU model agrees much better with that of the Euler equations than the HOU model, as noted previously. In addition, as $\mu_{21}<0$ for all $k$, the wave speed $c$ is always positive, which is not the case for the HOU model. In figure $3(b)-(d)$, for a fixed value of $\rho_{2} / \rho_{1}=1.01$, the coefficients of the aHOU model are compared with those of the HOU model. The coefficients of the aHOU model vary with the depth ratio $h_{2} / h_{1}$ similarly to those of the HOU model, but the difference between the two models increases with the depth ratio.

\subsection{The Gardner equation with adjusted coefficients}

When $\mu_{4}=0$, the aHOU model can be reduced to

$$
\zeta_{t}+\mu_{0} \zeta_{x}+\mu_{1} \zeta \zeta_{x}+\mu_{21} \zeta_{x x x}-\mu_{22} \zeta_{x x t}+\mu_{3} \zeta^{2} \zeta_{x}=0
$$

or, equivalently,

$$
\left(\zeta-\mu_{22} \zeta_{x x}\right)_{t}+\left(\mu_{0} \zeta+\frac{1}{2} \mu_{1} \zeta^{2}+\mu_{21} \zeta_{x x}+\frac{1}{3} \mu_{3} \zeta^{3}\right)_{x}=0
$$

For simplicity, this equation will be hereafter referred to as the adjusted Gardner (aG) equation as it has both quadratic and cubic nonlinear terms. Due to the additional term with $\mu_{22}$, notice that the aG equation has different mathematical properties from the Gardner equation, including the loss of integrability (Olver 1979). With $\mu_{1}=c_{1}, \mu_{22}=0$, and $\mu_{3}=c_{3}$, the original Gardner equation (2.10) can be recovered from (3.15). When $\mu_{3}=0,(3.15)$ with (3.11) is the $\mathrm{KdV}$-BBM equation, which is asymptotically consistent with the KdV equation.

For traveling waves, the aG equation given by (3.16) can be easily reduced to

$$
\zeta_{X}^{2}=\bar{\gamma}_{0} \zeta^{2}\left(\zeta^{2}+\gamma_{1} \zeta+\gamma_{2}\right)
$$

where $\gamma_{1}$ and $\gamma_{2}$ are the same as those of the aHOU model given by (3.5) and $\bar{\gamma}_{0}$ is given by

$$
\bar{\gamma}_{0}=-\frac{\mu_{3}}{6\left(\mu_{21}+c \mu_{22}\right)} \text {. }
$$

As the steady form of the $\mathrm{aG}$ equation (3.17) has the same structure as that of the original Gardner equation, as

$$
\zeta_{\mathrm{aG}}(X)=\frac{a}{\bar{b}+(1-\bar{b}) \cosh ^{2}(\bar{K} X)}, \quad X=x-c_{\mathrm{aG}} t,
$$

where $\bar{b}$ are $\bar{K}$ given by

$$
\bar{b}=a^{2} / \gamma_{2}, \quad \bar{K}=\left(\bar{\gamma}_{0} \gamma_{2}\right)^{1 / 2} / 2 .
$$

Notice that the solitary wave speed of the aG equation, $c_{\mathrm{aG}}$, is identical to that of the aHOU model given by (3.6) while its effective wavelength is given, from (2.17), by

$$
\lambda_{\mathrm{aG}}=\frac{2}{|a| \bar{\gamma}_{0}^{1 / 2}} \tanh ^{-1} \frac{|a|}{\gamma_{2}^{1 / 2}} .
$$




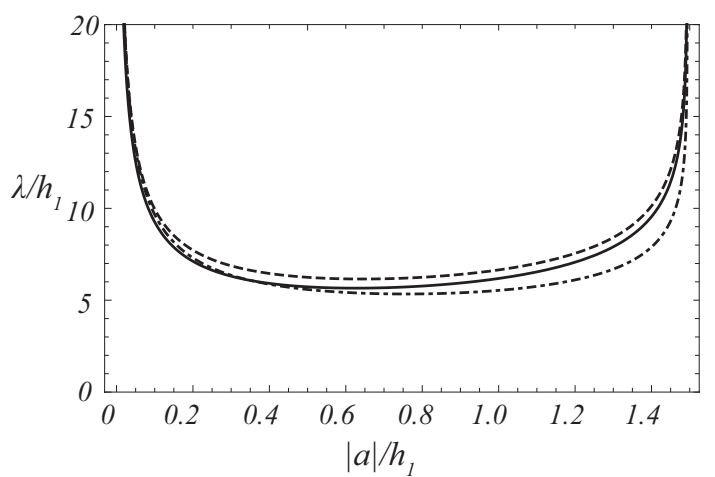

$(a)$

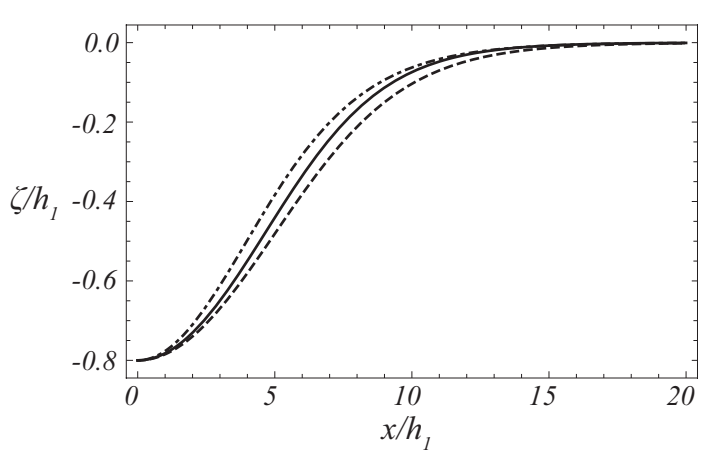

(c)

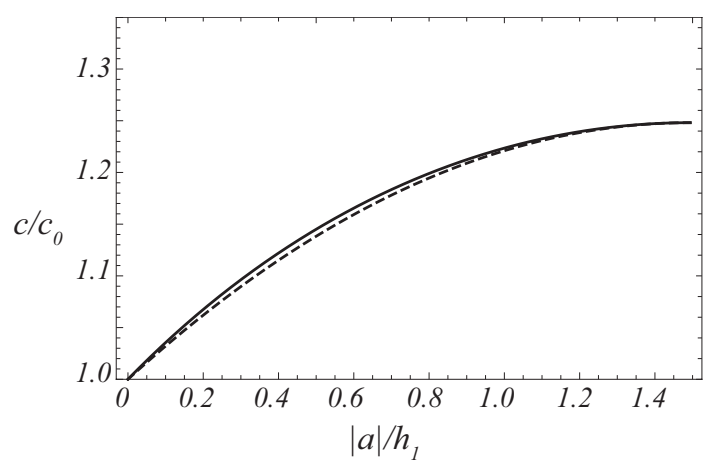

(b)

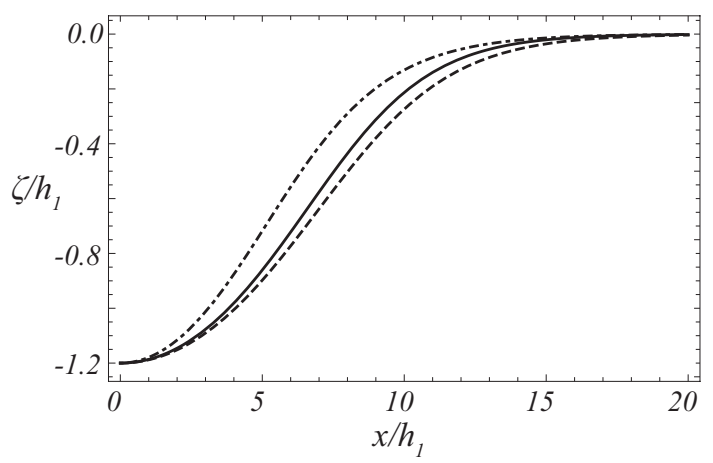

$(d)$

Figure 4: Comparison between the MCC (solid), aHOU (dashed), and aG (dot-dashed) for $\rho_{2} / \rho_{1}=1.01$ and $h_{2} / h_{1}=4$. (a) Effective wavelength $\left(\lambda / h_{1}\right)$ versus wave amplitude $\left(a / h_{1}\right)$. (b) Wave speed $\left(c / c_{0}\right)$ versus wave amplitude $\left(a / h_{1}\right)$. (c) Half solitary wave profiles of depression for $a / h_{1}=-0.8$. (d) Half solitary wave profiles of depression for $a / h_{1}=-1.2$, for which the original HOU and Gardner equations have no solitary wave solutions.

\subsection{Solitary wave solutions}

As shown in Figure 4(a), both the aHOU and aG models now predict the effective wavelength to the maximum wave amplitude for the MCC model. In particular, the effective wavelength for the aHOU equation agrees well with that for the MCC model over a wide range of wave amplitudes although it is slightly overestimated. On the other hand, the aG model underestimates the effective wavelength. This implies that the nonlinear dispersive term in the aHOU model is essential for the accurate prediction of the effective wavelength. As can be seen in Figure 4(b), the wave speed for the aHOU model is slightly smaller than the MCC wave speed over a range of intermediate wave amplitudes, but the difference is insignificant.

In figure $4(c)$, the solitary wave profiles are compared for $a / h_{1}=-0.8$. The original HOU and Gardner equations have the solitary wave solutions, but, the comparison with the MCC solution is poor, as shown in figure $2(b)$. On the other hand, the adjusted models clearly improve the comparison. Figure $4(d)$ shows the solitary wave solutions of the aHOU and aG models 


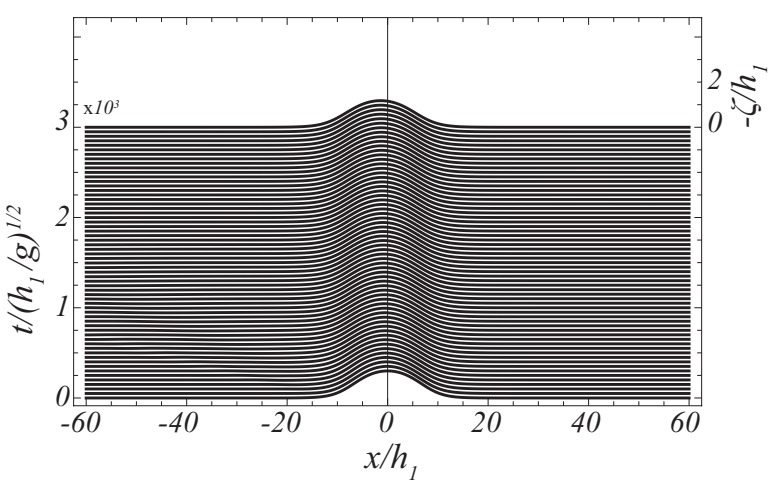

(a)

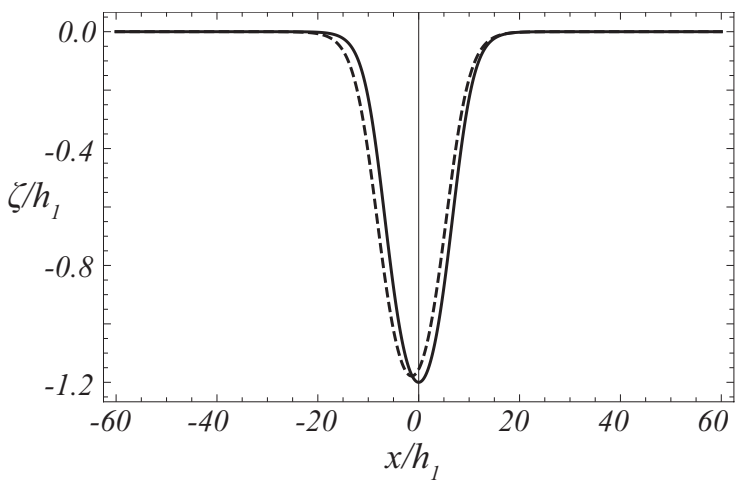

(b)

Figure 5: Numerical solutions of the aHOU initialized by a single MCC solitary wave of depression with $a / h_{1}=-1.2$ for $0 \leq t /\left(h_{1} / g\right)^{1 / 2} \leq 3000$. The solutions are presented in the reference frame moving with the speed of the MCC solitary wave. (a) Time evolution of $-\zeta / h_{1}$; (b) Wave profiles for the aHOU model (dashed) compared compared with the MCC solution at $t /\left(h_{1} / g\right)^{1 / 2}=3000$. Here the density and depth ratios are $\rho_{2} / \rho_{1}=1.028$ and $h_{2} / h_{1}=4$.

for $a / h_{1}=-1.2$. The comparison of the aHOU model with the MCC model is outstanding. It should be remarked that the original HOU and Gardner models have no solitary wave solutions for this amplitude.

\subsection{Numerical solutions for evolving solitary waves}

While the solitary wave solution of the aHOU model compares well with that of the MCC equations, its applicability to time dependent problems is still uncertain and needs to be tested. Here, we solve numerically the MCC, aHOU, and aG equations using a second-order central difference scheme in both space and time with zero and radiation boundary $\left(\zeta_{t}+c_{0} \zeta_{x}=0\right)$ conditions at the left and right boundaries, respectively, as described in Jo \& Choi (2002). As discussed in Jo \& Choi (2008), to eliminate the local Kelvin-Helmholtz instability from the MCC model (Jo \& Choi 2002), a low-pass filter that eliminates short waves whose wavenumbers are greater than criticality has been applied when the MCC model is solved numerically. With the number of spatial grid points $N=2^{11}$, and $\Delta t /\left(h_{1} / g\right)^{1 / 2}=0.005$, when the aHOU model is initialized with its own solitary wave solution, the relative errors in conserving excess mass and energy are found to be $10^{-5}$ and $10^{-8}$, respectively, at $t=3 \times 10^{3}$. Therefore, the numerical scheme is considered reliable. It should be also remarked that no filter has been applied when the aHOU model is solved while the MCC model cannot be solved for large amplitude internal waves without a filter for the spatial resolution used here. In addition, no initial conditions for the velocity fields are necessary for the aHOU model.

To confirm that the aHOU model behaves similarly to the MCC model even for unsteady problems, we first consider a simple problem of the propagation of a single solitary wave of the MCC model, whose wave amplitude is $a / h_{1}=-1.2$. To address the importance of the nonlinear 


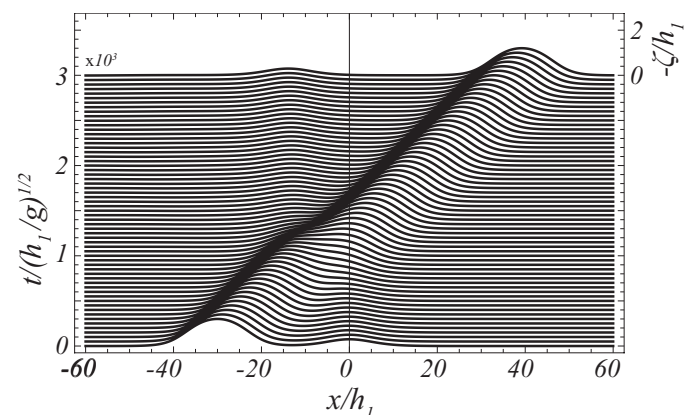

(a)

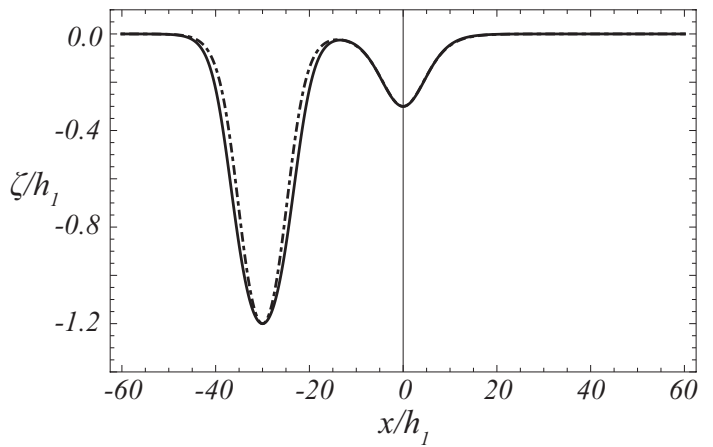

$(c)$

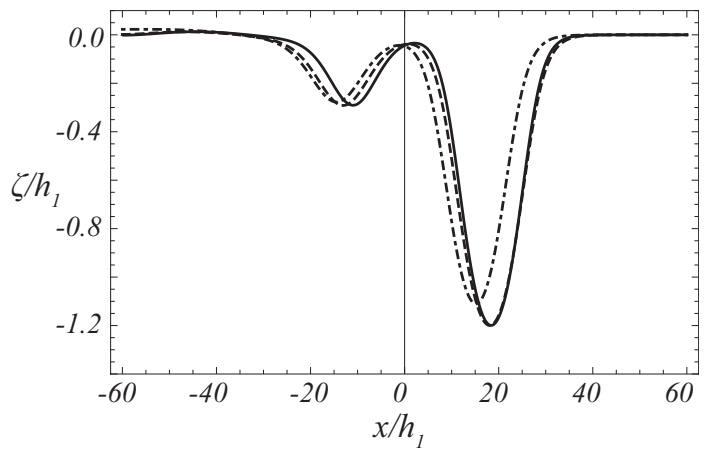

(e)

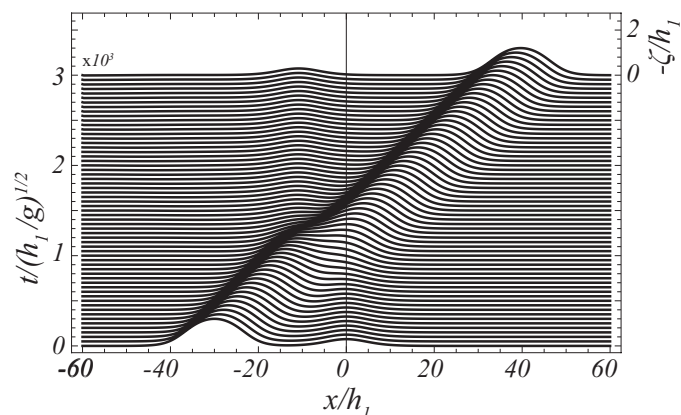

(b)

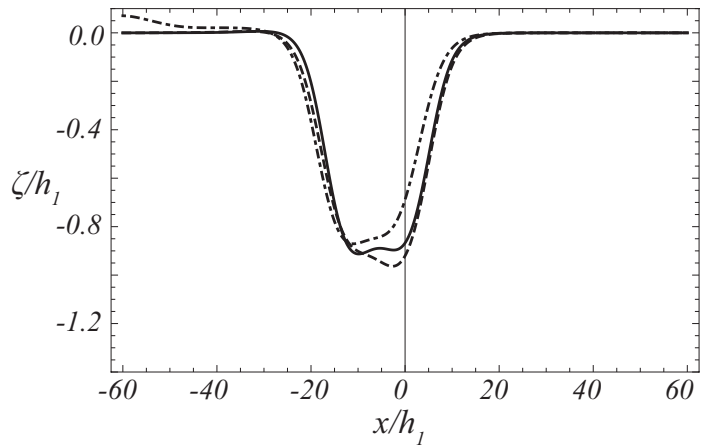

(d)

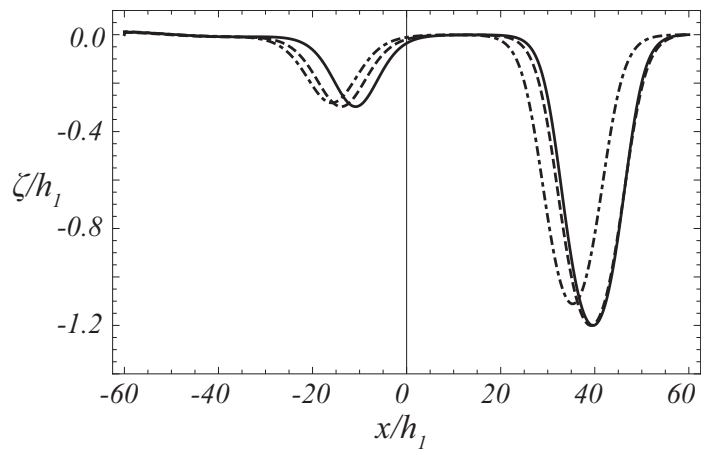

$(f)$

Figure 6: Comparison between MCC (solid), aHOU (dashed), and aG (dot-dashed) equations for the overtaking collision between two solitary waves of depression with $a / h_{1}=-1.2$ and $a / h_{1}=-0.3$ for $0 \leq t /\left(h_{1} / g\right)^{1 / 2} \leq 3000$. The models are initialized with their own solitary waves and are solved in a frame of reference moving with the solitary wave of $a=-0.3$. (a) Numerical solutions of MCC; (b) Numerical solutions of aHOU; $(c) t /\left(h_{1} / g\right)^{1 / 2}=0 ;(d)$ $t /\left(h_{1} / g\right)^{1 / 2}=1000 ;(e) t /\left(h_{1} / g\right)^{1 / 2}=2000 ;(f) t /\left(h_{1} / g\right)^{1 / 2}=3000$. Here the density and depth ratios are $\rho_{2} / \rho_{1}=1.028$ and $h_{2} / h_{1}=4$. 
dispersive term, the aG model is also solved numerically. As the MCC solitary wave is not a solution of either unidirectional model, it is expected to be initially deformed, but the numerical solution should remain close the initial wave profile as the solitary wave solution of each model approximates reasonably well that of the MCC model. Notice that the numerical solutions in figure 5 are presented in a frame of reference moving with the speed of the MCC solitary wave. As the solitary wave solution of the aHOU model is closer to the MCC solitary wave than the aG model, smaller dispersive waves are generated initially for the aHOU model, but propagate downstream to disappear from the computational domain. At $t /\left(h_{1} / g\right)^{1 / 2}=3000$, the two models show almost steady-state profiles although the aHOU solution is slightly smaller and slower than the aG solution. Considering the effective wavelength of the aG model is smaller than that of the MCC model and the mass lost to the dispersive tail is small, one can expect that the wave amplitude of the steady-state solution of the aG model is greater than the initial amplitude.

Next we consider numerically overtaking collision of two solitary waves, whose amplitudes are $a / h_{1}=-1.2$ and $a / h_{1}=-0.3$. Here each model is initialized with its own solitary wave solutions. This would test if the adjusted unidirectional models are reliable theoretical tools for time dependent problems in comparison with the MCC model. Figure $6(a)$ and $(b)$ show the numerical solutions of the aHOU and aG models, respectively, for $0 \leq t /\left(h_{1} / g\right)^{1 / 2} \leq 3000$. Both solutions show little disturbances, except for a phase shift after the overtaking collision. The detailed comparison of numerical solutions at $t /\left(h_{1} / g\right)^{1 / 2}=0,1000,2000,3000$ is made in figure $6(c-f)$. As shown in figure $6(c)$, the initial solitary wave solution of the aHOU model is close to the MCC while that of the aG model is slightly narrower than the others. As shown in figure $6(f)$, after the collision, the taller wave of the aHOU model is still located close to that of the MCC model although the smaller wave experiences a greater phase shift. On the other hand, the amplitude of the taller wave of the $\mathrm{aG}$ model is reduced and stays behind the taller waves of the other models. Based on this comparison, it can be concluded that the mHOU model better represents the MCC model than the aG model and, therefore, only the aHOU model is considered in the following discussion for variable bottom.

\section{Propagation over variable bottom}

The propagation of weakly nonlinear long internal waves in a two-layer system with variable bottom can be modeled by the $\mathrm{KdV}$ equation with variable coefficients (Djordjevic \& Redekopp, 1978; Jo \& Choi, 2012):

$$
\zeta_{t}+c_{0}(x) \zeta_{x}+c_{1}(x) \zeta \zeta_{x}+c_{2}(x) \zeta_{x x x}+\frac{1}{2} c_{0}^{\prime}(x) \zeta=0,
$$

where $c_{i}(x)(i=0,1,2)$ can be found with replacing $h_{2}$ in (2.5) and (2.8) by the local lower-layer thickness, $h_{2}(x)$, and $c_{0}^{\prime}(x)=\mathrm{d} c_{0} / \mathrm{d} x$. Notice that the depth variation is assumed to be slow, or more specifically, $h_{2}^{\prime}(x)=O\left(\epsilon^{3}\right)$.

A similar approach can be applied to the aHOU model, which yields the following aHOU equation with variable coefficients:

$\zeta_{t}+\mu_{0}(x) \zeta_{x}+\mu_{1}(x) \zeta \zeta_{x}+\mu_{21}(x) \zeta_{x x x}-\mu_{22}(x) \zeta_{x x t}+\mu_{3}(x) \zeta^{2} \zeta_{x}+\mu_{4}(x)\left(\zeta_{x}^{2}+2 \zeta \zeta_{x x}\right)_{x}+\mu_{5}(x) \zeta=0$. 
Here, as in (4.1), the expressions of $\mu_{i}(x)$ except for $i=5$ can be found from those of $\mu_{i}$ chosen in $\S 3.1$ with replacing $h_{2}$ by the local lower layer thickness $h_{2}(x)$. The expression of $\mu_{5}(x)$ can be adjusted to better describe the deformation of large amplitude internal solitary waves propagating over variable bottom, but is chosen here as $\mu_{5}(x)=c_{0}^{\prime}(x) / 2$, by following (4.1), as the leading-order approximation. It should be noticed that, if necessary, one can choose $\mu_{5}(x)=c_{0}^{\prime}(x) / 2+\nu$, where $\nu>0$ is the damping coefficient representing viscous energy dissipation due to bottom friction although it should be measured or modeled.

To validate the aHOU model with variable coefficients, we solve the MCC model $(i=1,2)$ for variable bottom given (Jo \& Choi 2002) by

$$
\eta_{i t}+\left(\eta_{i} \bar{u}_{i}\right)_{x}=0, \quad \bar{u}_{i t}+\bar{u}_{i} \bar{u}_{i x}+g \zeta_{x}=-\frac{P_{x}}{\rho_{i}}+\frac{1}{\eta_{i}}\left(\frac{1}{3} \eta_{i}{ }^{3} G_{i}\right)_{x}+B_{i},
$$

where $\eta_{2}=h_{2}(x)+\zeta, B_{1}=0$, and $B_{2}$ is given by

$$
B_{2}=\frac{1}{\eta_{2}}\left(\frac{1}{2} \eta_{2}^{2} H_{2}\right)_{x}-\left(\frac{1}{2} \eta_{2} G_{2}+H_{2}\right) h_{2}^{\prime}(x),
$$

with $H_{2}$ defined by

$$
H_{2}=\left(\partial_{t}+\bar{u}_{2} \partial_{x}\right)\left(h_{2}^{\prime} \bar{u}_{2}\right) .
$$

The remaining variables are the same as before, except for $\bar{u}_{2}$, which is now the horizontal velocity averaged over the slowly-varying local thickness of the lower layer. If the depth variation is so slow that $h_{2}^{\prime}(x)=O\left(\epsilon^{3}\right)$, one can assume $B_{2}=0$ without violating the asymptotic assumption to derive the MCC model. For a single layer $\left(\rho_{1}=0\right)$, under the weakly nonlinear assumption, (4.3) can be reduced to the model of Peregrine (1967) for surface waves.

To test the aHOU for variable bottom, we study numerically the propagation of a single solitary wave over an isolated bottom topography of finite amplitude given by

$$
b(x)=b_{0} \cos ^{2}(4 \pi / L) \quad \text { for }|x| \leq L / 8,
$$

where $b_{0} / h_{1}=2.4 ; b(x)=0$ for $|x|>L / 8$. Then the local thickness of the lower layer is given by $h_{2}=h_{20}-b(x)$, where $h_{20}$ is the undisturbed thickness of the lower layer. Here the total computational domain $L$ is chosen $L / h_{1}=240$ and the depth and density ratios are given by $\rho_{2} / \rho_{1}=1.028$ and $h_{20} / h_{1}=4$, respectively. Initially a single solitary wave of $a / h_{1}=-1.2$ is located at $x / h_{1}=-60$ and both models are initialized with their own solitary wave solutions. Once again, this amplitude is greater than the maximum wave amplitude of the original HOU equation.

Figure 7 shows the time evolution of a single solitary wave for $0 \leq t /\left(h_{1} / g\right)^{1 / 2} \leq 800$ as it passes through the topography given by (4.6). As the MCC model describes bi-directional waves, one can observe in figure 7 $(a)$ both the deformed solitary wave propagating in the positive $x$-direction and small-amplitude reflected waves propagating in the opposite direction. On the other hand, as shown in figure $7(b)$, the aHOU model describes only the waves traveling to the right. Considering that the aHOU model is unable to capture the reflected waves, when the two numerical solutions are compared in figure $7(c-f)$, their agreement is surprising, in particular, in terms of the evolution of the leading solitary wave. However the linear dispersive tails behind the leading wave are different. It should be remarked that the linear dispersion relations of the two models are different and the filter applied to the MCC model to eliminate local instability also modifies the dispersive behavior of relatively short waves. 


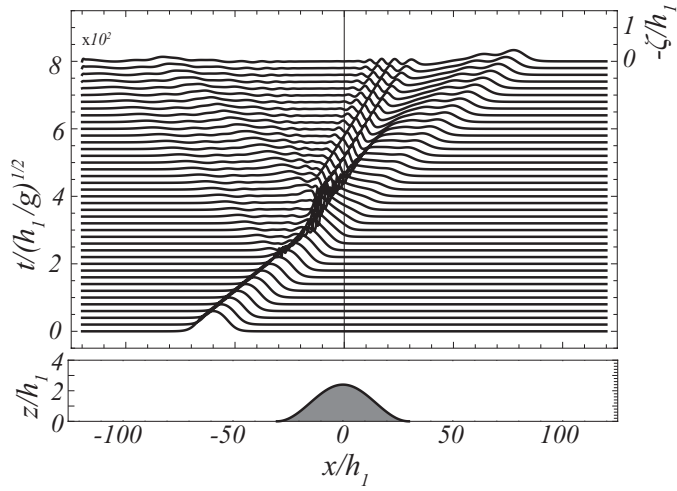

(a)

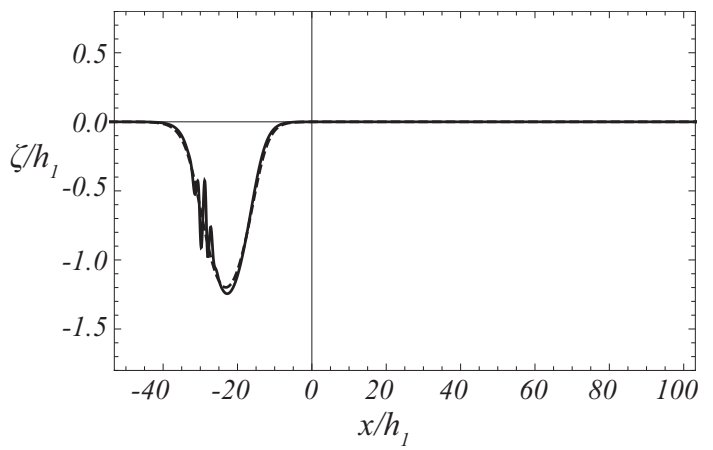

$(c)$

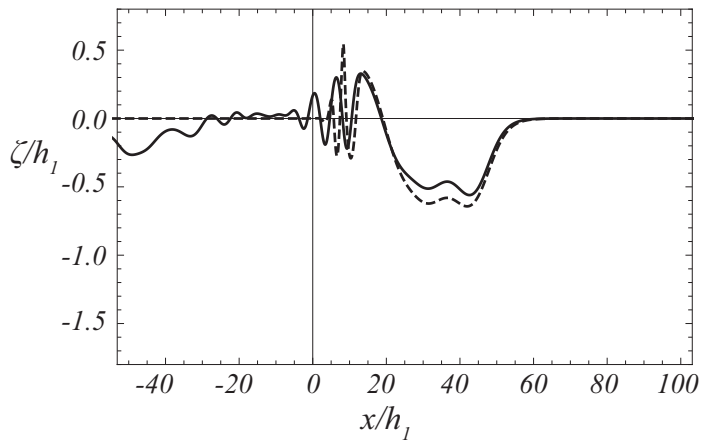

$(e)$

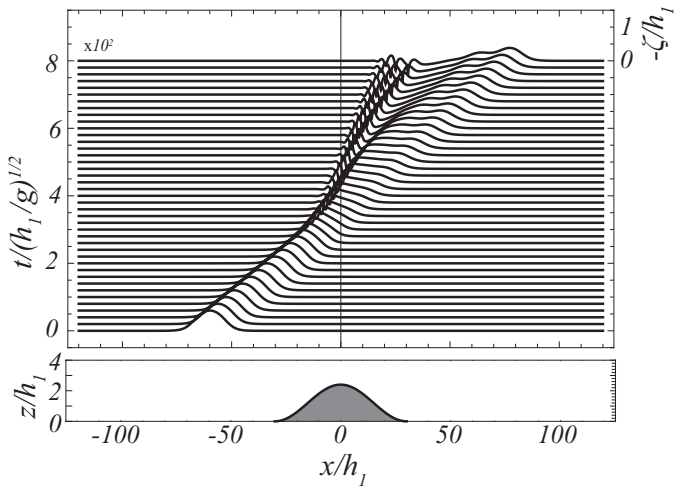

(b)

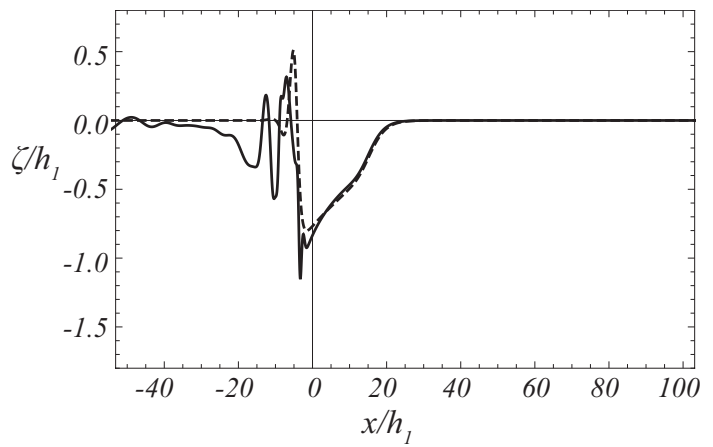

(d)

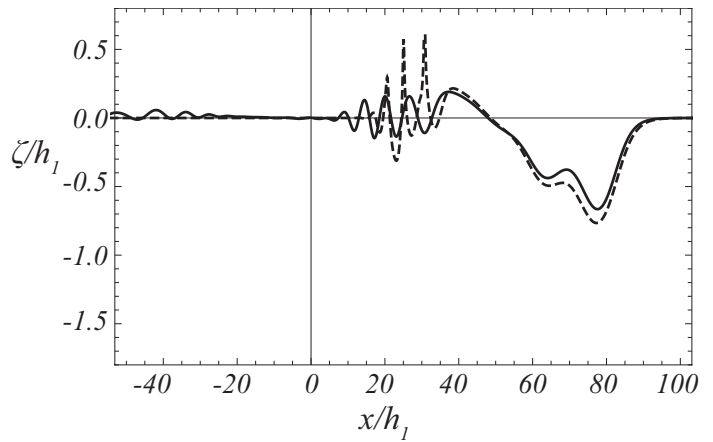

$(f)$

Figure 7: Comparison between the MCC (solid) and aHOU (dashed) models for the propagation of a single solitary wave of depression with $a / h_{1}=-1.2$ over an isolated bottom topography given by (4.6) for $0 \leq t /\left(h_{1} / g\right)^{1 / 2} \leq 800$. The models are initialized with their own solitary wave solutions located at $x / h_{1}=-60$. (a) Numerical solutions of MCC; $(b)$ Numerical solutions of aHOU; $(c) t /\left(h_{1} / g\right)^{1 / 2}=200 ;(d) t /\left(h_{1} / g\right)^{1 / 2}=400 ;(e) t /\left(h_{1} / g\right)^{1 / 2}=600 ;(f) t /\left(h_{1} / g\right)^{1 / 2}=$ 800. Here the density and depth ratios are $\rho_{2} / \rho_{1}=1.028$ and $h_{20} / h_{1}=4$. 


\section{Conclusion}

It is shown that the high-order unidirectional model originally derived for weakly nonlinear waves can describe internal solitary waves of large amplitudes when the coefficients are adjusted appropriately. Using the fact that the mathematical structure of the steady HOU model is similar to that of the MCC equations, the coefficients for the second and third-order nonlinear and nonlinear dispersive terms are adjusted to reproduce the solitary wave solution of the MCC model as closely as possible. When compared with the MCC model, it is found that the aHOU model well describes not only the solitary wave profiles up to the maximum wave amplitude, but also their dynamics even when they propagate over variable bottom. It should be stressed that the original HOU model is asymptotically consistent, but fails to describe such large amplitude waves.

The aHOU model is simple and convenient for numerical studies. Contrary to the MCC model (and also the Euler equations), it requires no numerical filter and no initial conditions for the horizontal velocities. Furthermore, the coefficients of the aHOU model depend only the physical parameters (the depth and density ratios) and contains no parameters to be tuned. Therefore, one can conclude that the aHOU equation can serve as a useful theoretical model to study large amplitude internal solitary waves and can be applied to a wide range of practical problems.

\section{Acknowledgements}

WC gratefully acknowledges support from the US National Science Foundation through Grant No. OCE-1634939. CZ acknowledges support from the China Scholarship Council for her one-year visit to NJIT in 2018-2019.

\section{References}

[1] Camassa, R., Choi, W., Michallet, H., Rusas, P. \& Sveen, J. K. 2006 On the realm of validity of strongly nonlinear asymptotic approximations for internal waves, J. Fluid Mech. 549, 1-23.

[2] Choi, W. \& Camassa, R. 1999 Fully nonlinear internal waves in a two-fluid system, J. Fluid Mech. 396, 1-36.

[3] Choi, W., Barros, R., Jo, T.-C. 2009 A regularized model for strongly nonlinear internal solitary waves. J. Fluid Mech. 629, 73-85.

[4] Choi, W., Goullet, A., \& Jo, T.-C. 2011 An iterative method to solve a regularized model, for strongly nonlinear long internal waves. J. Comp. Phys. 230, 2021-2030.

[5] Craig, W. \& Sternberg, P. 1992 Symmetry of free-surface flows. Arch. Ration. Mech. Anal. 118, 1-36. 
[6] Djordjevic, V. D. \& Redekopp, L. G. 2006 The fission and integration of internal solitary waves moving over two-dimensional topography. J. Phys. Oceanogr. 8, 1016-1024.

[7] Grimshaw, R.H.J., Pelinovsky, D., Pelinovsky, E. \& Slunyaev, A. 2002 Generation of largeamplitude solitons in the extended Korteweg-de Vries equation. Chaos 12, 1070-1076

[8] Grue, J., Jensen, A., Rusas, P.-O. \& Sveen J. K. 1999 Properties of large-amplitude internal waves. J. Fluid Mech. 380, 257-278.

[9] Helfrich, K. R. \& Melville, W. K. 2006 Long nonlinear internal waves. Annu. Rev. Fluid Mech. 38, 395-425.

[10] Jo, T. \& Choi, W. 2002 Dynamics of strongly nonlinear solitary waves in shallow water. Stud. Appl. Math. 109, 205-228.

[11] Jo, T. and Choi, W. 2008 On stabilizing the strongly nonlinear internal wave model. Studies in Applied Math. 120, 65-85.

[12] Kodaira, T., Waseda, T., Miyata, M. \& Choi, W. 2016 Internal solitary waves in a two-fluid system with a free surface. J. Fluid Mech. 804, 201-223.

[13] Kakutani, T. \& Yamasaki 1978 Solitary waves on a two-layer fluid. J. Phys. Soc. Japan 45, 674-679.

[14] Koop, C.G. \& Butler, G. 1981 An investigation of internal solitary waves in a two-fluid system. J. Fluid Mech. 112, 225-251.

[15] Michallet, H., \& Barthélemy, E. 1998 Experimental study of interfacial solitary waves. $J$. Fluid Mech. 366, 159-177.

[16] Miyata, M. 1988 Long internal waves of large amplitude. In H. Horikawa, H. Maruo, (Eds.), Proc. of the IUTAM Symp. on Nonlinear Water Waves, 399-406. miyata

[17] Olver, P. J. 1979 Euler operators and conservation laws of the BBM equation. Math. Proc. Camb. Phil. Soc. 85, 143-160.

[18] Ostrovsky, L. A. \& Grue, J. 2003 Evolution equations for strongly nonlinear internal waves. Phys. of Fluids 15, 2934-2948.

[19] Peregrine, D. H. 1967 Long waves on a beach. J. of Fluid Mech. 27, 815-827. 